\title{
The Impact of Burnout on the Academic Achievement of Saudi Female Students Enrolled in the Colleges of Health Sciences
}

\author{
Alanoud M Alshobaili ${ }^{1}$, Hadeel A Alshallan ${ }^{1}$, Shahad H Alruwaili ${ }^{1}$, Amal F Alqarni ${ }^{1}$, Maha M Alanazi ${ }^{1}$, Tmadhur A \\ Alshinqeeti ${ }^{1} \&$ Salwa S Awad ${ }^{2}$ \\ ${ }^{1}$ Students of Clinical Psychology, Department of Health Science, College of Health and Rehabilitation Sciences, \\ Princess Nourah Bint Abdulrahman University, Saudi Arabia \\ ${ }^{2}$ Assistant Professor of Psychology, Department of Rehabilitation Sciences, College of Health and Rehabilitation \\ Sciences, Princess Nourah Bint Abdulrahman University, Saudi Arabia \\ Correspondence: Salwa S Awad, PhD, Assistant Professor of Psychology, Department of Rehabilitation Sciences, \\ College of Health and Rehabilitation Sciences, Princess Nourah Bint Abdulrahman University, Riyadh, Saudi Arabia.
}

Received: October 2, 2020

doi:10.5430/ijhe.v10n2p229
Accepted: December 2, 2020

Online Published: December 8, 2020

\begin{abstract}
Objectives: The objectives of this study were to investigate the impact of burnout on the academic achievement of female students in the colleges of health sciences and to explore on their educational levels of study regarding burnout.

Methods: Purposive sampling technique was used in this study and a sample size of 540 Saudi female students was chosen to participate in this study. The tools used were Maslach Burnout Inventory-General Survey for Students MBIGS (S) to measure the level of burnout and a socio-demographic questionnaire were administered on participants. The MBI-GS (S) proved internal consistency reliability at 0.86 across many samples.

Results: The level of burnout among participants was $51.9 \%$. Some $27.2 \%$ of the participants experienced mild burnout, while $14.4 \%$ showed moderate levels of burnout. Only $6.5 \%$ of participants displayed a high level of burnout, and they were among the Colleges of Pharmacy (25.0\%) and Dentistry (10.1\%).

Conclusions: The study findings showed no association between burnout and academic achievement and no relationship between burnout and the academic level of study. Only the students of the College of Pharmacy suffered from high levels of burnout.
\end{abstract}

Keywords: PNU, colleges of health sciences, Maslach Burnout Inventory-General Survey, academic achievement

\section{Introduction}

Burnout was first addressed in 1974 by Herbert Freudenberger, who conducted several studies, which popularised the term (Heinemann \& Heinemann, 2017). The symptoms may vary from one person to the other. However, there are both physical symptoms and behavioural signs associated with burnout. The physical symptoms may include headache, exhaustion, loss of energy, dyspnoea, gastrointestinal issues and insomnia, while behavioural signs include distress, signs of depression, a sense of exhaustion caused by significant stress, anger and suspicious behaviours (Freudenberger, 1974, p. 161). Burnout syndrome has received a great deal of attention in the past decade. International studies have established a correlation between burnout syndrome and a number of circumstances, such as stress levels, clinical depression, job demands and environmental stressors. A study done by Schonfeld and Bianchi (2016) has shown that $86 \%$ of teachers diagnosed with depression have shown high burnout levels. Studies showed that students facing regular burnout symptoms would be incompetent and unwilling to exert effort on their academic duties. Therefore, this little effort will probably hinder the academic achievement (Richardson et al. 2012). Studies have been conducted in the Kingdom of Saudi Arabia (KSA) investigating burnout syndrome, most of them mainly focussing on medical and dental students (Aboalshamat et al., 2017), physicians (Aldrees et al., 2013), nurses (Al-Turki et al., 2010) and physiotherapists (Al-Imam \& Al-Sobayel, 2014), while a few researchers have addressed other fields. A study have shown that $65.9 \%$ of intensive care nurses working in King Saud Medical City were reported to have high levels of burnout (Awajeh, Issa, Rasheed \& Faisal Amirah, 2018). Furthermore, a systematic review was done in the KSA to examine burnout syndrome among different health specialists and reported that there were significant levels of burnout among health care specialists (doctors, nurses and physiotherapy) in Arab Countries (Elbarazi, Loney, Yousef \& Elias, 2017). Study conducted by Almalki, et al. (2017) indicated that $67 \%$ of medical students in the KSA suffer from 
burnout. In recent years, there have been studies, which demonstrated the effect of burnout syndrome among medical students at different stages of study, and it has been shown that the levels of burnout increase as students progress in their education (Asencio-López et al., 2016). A recent study by Alyamani et al. (2018) has shown that senior residents at King Abdulaziz Medical City in Riyadh were more likely to experience burnout than junior residents. However, there was no association between burnout and extracurricular activities among students (Alyamani et al., 2018). It is important to understand the effects of burnout syndrome on students. This will help students and faculty members to be aware of the signs and symptoms of burnout syndrome so that strategies and plans can be developed to help students improve their academic performance. It is also important to address the relationship between burnout and students' academic achievement in order to help students deal with stress related to their learning process. Students are at high risk of developing burnout because of the pressure of their studies, particularly health sciences students who are studying medicine, nursing, allied health sciences, pharmacy and dentistry. Burnout symptoms also affect student's academic achievement and motivation to study (Salmela-Aro, Tolvanen \& Numri, 2009; cited in Cazan, 2015).

According to a study by Al Ubaidi, Jassim and Salem (2018), one of the struggles medical students face is the pressure associated with balancing academic life and social life. Moreover, other studies have shown that students of majors other than medicine, who do not have a coping mechanism to deal with the study load and stress, may be more susceptible to substance abuse (Seo et al., 2015). They may have unhealthy habits resulting from reduced physical activity or no previous practice of physical activity at all (Seo et al., 2015; Fares et al., 2016; Dafaalla, 2016; De Oliva Costa et al., 2012; Kulsoom, 2015). However, most of the statistics found related to medical students in Arab countries show various levels of stress, with 53-72\% of medical students in Saudi Arabia experiencing stress, 60\% in Egypt experiencing stress and 50\% in Sudan experiencing stress, while the reported prevalence of burnout was between 10\% and 45\% (Al Ubaidi, Jassim \& Salem, 2018). A study conducted by Kroska, Calarge, O'Hara, Deumic and Dindo (2017) has shown that depression and work stress are linked to burnout syndrome. Cofer et al. (2018) emphasised the role of emotional intelligence in burnout syndrome: high levels of emotional intelligence were shown to have a positive correlation with low burnout syndrome. Moreover, a study conducted by Wood (2017) showed that students who study in centralised locations have low levels of burnout and stress compared with students who study in decentralised locations. This might be due to access to more opportunities to socialise to relieve stress, and have access to mental health care, etc.

Saudi Arabia is a socially conservative country with a combination of culture and Islamic religion. This unique mixture and the country's cultural homogeneity pose difficulties for Saudi females. For example, Saudi women until recently were not allowed to practise law or engineering, nor were they allowed to drive or travel alone. In recent years, the Saudi government has opened up some of these practices for women, but the issue remains in some Saudi families, in which the belief that women should be forbidden from such practices still exists. These difficulties are not a part of Islam or Sharia law but are cultural beliefs, which have been forced in the Saudi society. The barriers and limitations Saudi women face not only pose difficulties for women but also affect the country's functioning and development (Al Alhareth, Al Alhareth and Al Dighrir, 2015).

The limitations and cultural barriers Saudi females face may be considered risk factors which might add to their stress levels and result in an increase in burnout syndrome among females. A recent study conducted among Saudi Medical Residency staff, which investigated duty hours, and burnout syndrome found that female gender was associated with a significantly high prevalence of burnout compared with male resident staff. A study conducted by Alwazzan and Rees (2016) investigated cultural attitudes regarding females in medical education and concluded that the inequality between genders in cultural perceptions was significant in Saudi Arabia compared with western countries: for example, the limitations on female independence, decision-making and leadership positions. In addition, Al-bakr, Bruce, Davidson, Schlaffer and Kropiunigg (2017) have asserted that $64 \%$ to $76 \%$ of university students in Saudi Arabia recognised that females receive less support than males regarding their education and employment. Social barriers, such as transportation difficulties, early marriages and the requirement for the male's permission for females to engage in certain practices, influence females' decisions regarding whether to continue or discontinue their studies in higher education (Al Alhareth, Al Dighrir \& Al Alhareth, 2015). The significantly high results in burnout among females has been explained by high parental expectations, greater cultural pressure and low social exposure (Abdelghani et al., 2011; Abdelrahman et al., 2013; El-masry, 2013, cited in Al Ubaidi, Jassim \& Salem, 2018; Fawsy, 2017; Paro et al., 2014). Another cross-sectional study conducted in Saudi Arabia that investigated the effects of stress on medical students found that high levels of psychological stress were associated with female students at a greater degree than in male students. The effect of education level on burnout was significantly high in the initial three years of study in medical school compared with the last two years (Abdulghani, 2019). Therefore, this current study is aiming to 
investigate the impact of burnout on the academic achievement of female students' who strive to prove themselves in the field of science specialities.

\section{Data and Methodology}

The present study adopted a purposive sampling technique, as the target population was female students who study in the health science colleges. The study took place at Princess Nourah bint Abdulrahman University (PNU), which is located in Riyadh City in the Kingdom of Saudi Arabia and is considered the largest women's university in the world. The university, was founded in 1970 as the first college of education for women in the KSA, then renamed as Princess Nourah bint Abdulrahman University, and officially opened in 2008 (www.pnu.edu.sa. Accessed December 2019). The study participants were female students enrolled in PNU, particularly students majoring in health sciences namely, the College of Health and Rehabilitation Sciences, the College of Medicine, the College of Dentistry, the College of Nursing and the College of Pharmacy. The study was conducted using self-administered questionnaires for data collection for this study were intended to describe individual characteristics of the participants, their exposure to burnout, and information about the outcomes.

Inclusion criteria was considering the female students of the health colleges at PNU. Students diagnosed with depression or bipolar or anxiety disorders were excluded. Students in the foundation year were also excluded from the study as they are receiving their basic education and are not yet specialised in any of the previously mentioned specialties.

\subsection{Sampling Procedure}

A stratified sampling technique was employed. In the first stage, the sample size was divided between all health colleges by probability proportional to size as follows:

\begin{tabular}{lll} 
Table 1. Study Sample & & \\
\hline College & $\begin{array}{l}\text { Sample } \\
\text { Calculation }\end{array}$ & Total \\
\hline Health \& Rehabilitation Sciences & $687 / 2455 \times 500$ & 140 \\
Nursing & $601 / 2455 \times 500$ & 122 \\
Pharmacy & $586 / 2455 \times 500$ & 120 \\
Medicine & $382 / 2455 \times 500$ & 78 \\
Dentistry & $199 / 2455 \times 500$ & 40 \\
Total sample size & & 540
\end{tabular}

The second stage involved the selection of the clusters. One cluster was randomly chosen from each level from each college and from each program at the time of data collection; the clustering was done to obtain knowledge about the level of burnout burden on students according to their study specialisation and to measure whether the burnout level is affecting students' academic achievement. For this study, researchers have used purposive random sampling technique. The sample was selected from among students at three levels in each college: beginners, intermediate and advanced level. The study total sample size was 540 Saudi female students based on the mean of the health colleges.

\subsection{Data Collection}

The independent variable in this study was burnout among students, and the dependent variable was their academic achievement. The variables were measured using the Maslach Burnout Inventory-General Survey for Students MBIGS (S) (Kitaoka-Higashiguchi et al., 2004). The MBI-GS (S) is used to measure the levels of burnout in students and is a valid instrument, which has been widely used, in academic research. An Arabic version of the MBI inventory was used for the present study in order to have a reliable instrument for data analysis (Zaki, 2016). The researchers of the present study have developed the socio-demographic questionnaire that contained questions requesting personal information, such as age, gender, marital status and educational information that specifically enquiring about specialty, academic level and grade point average (GPA).

The data were collected through self-administered questionnaires, which were prepared using an online Google form to be completed electronically. An electronic link was distributed to students in all health sciences colleges through each class leader to guarantee that participants could immediately begin filling out the electronic questionnaires. Hard copies of the questionnaires were also prepared for any complications participants might encounter with the electronic copy, such as a weak internet signal, the lack of a laptop or an iPad, or any other issue. The researchers were very keen 
to identify eligible participants based on the study's inclusion and exclusion criteria; afterwards, the participants were randomly selected.

All statistical analyses were done using Statistical Package for the Social Sciences (SPSS, V. 24) software (IBM, 2016). Data were analysed to measure the level of burnout and its impact on the academic performance of students in health colleges. Since the researchers were looking for associations between burnout categories and academic performance ratio variables, data were represented in the descriptive part of the analysis by frequencies and percentages and in the inferential part by chi-square test results for all the variables as well as the ANOVA for data analysis.

Cross tabulation was used to analyse the relationship between variables at the level of the sample, and the chi-square test was used to infer at the population level. The data was considered statistically significant when the p-value was $<0.05$.

\subsection{Ethical Considerations}

All procedures performed in this study were done in accordance with the ethical standards of the institutional and/or national research committee. All participants were provided with a document that stated the nature and purpose of the study, giving informed consent. The university's Institutional Research Board (IRB) ethical approval was obtained prior to the commencement of the study. All the participants were assured that their personal information would not be identifiable, and participants were not asked to reveal their personal identity when they logged on to fill out the questionnaire to confirm participants' confidentiality. They were also given an assurance that the data collected will be stored in a secure manner on laptops, which would only be used for the purpose of research. The informed consent was signed and received from each participant before joining in the study.

\section{Results}

\subsection{Demographic Characteristics}

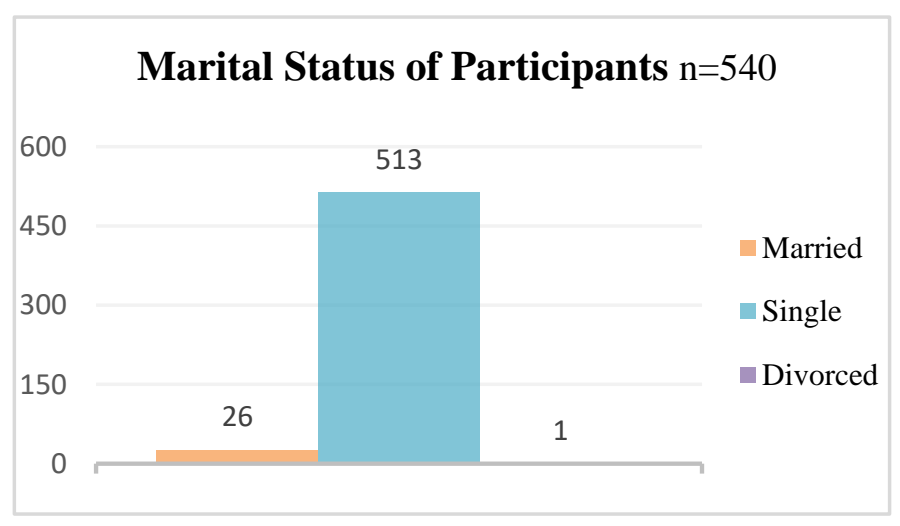

Figure 1. Marital status of participants.

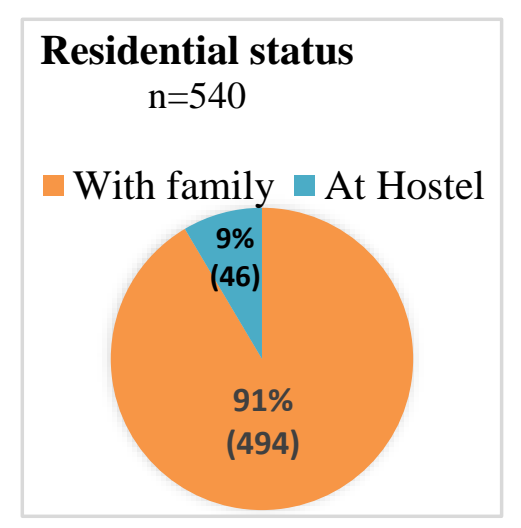

Figure 2. Residence of participants.

The age of all the participants of this study ranged between 19 and 26 years of age. The majority of participants (51\%) were aged 22 to 23, which is the university age of most of university students in Saudi Arabia. While the least common 
age group was 26 or older $(0.2 \%)$. The participants' marital status was divided into three categories. Only $4.8 \%$ of the sample were married, while $95 \%$ of the participants were single, which represents most of the participants (see Figure 1). Figure 2 shows the residential status of the participants. Only $8.5 \%$ of the participants live in the university hostel, while the rest $(91 \%)$ reside in their families' homes.

\subsection{Academic Characteristics}

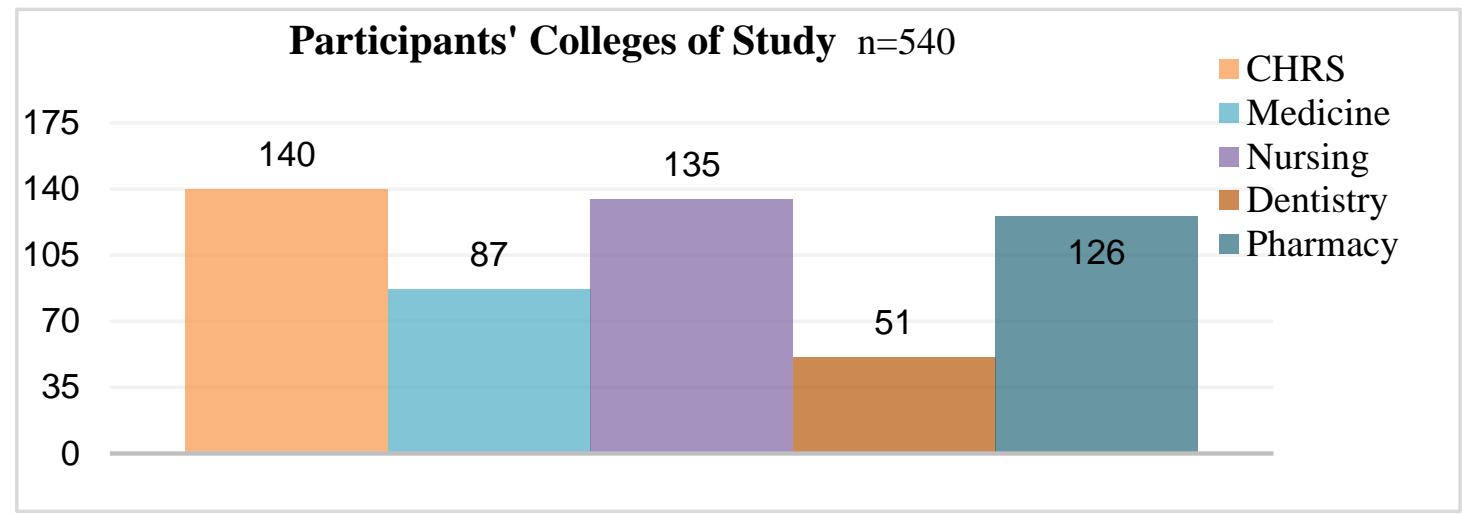

Figure 3. The colleges in which participants study.

The sample was collected from five colleges: 140 students from the College of Health and Rehabilitation Sciences, which represents the highest percentage $(25.9 \%)$ of study participants; 87 students from the college of medicine (16.1\%); 135 students from the college of nursing (25\%); 51 students from the college of dentistry (9.5\%); and 126 students from the college of pharmacy (23.4\%).

\subsection{Burnout among Participants}

\section{Participants' Level of Burnout $n=540$}

- Normal No Burnout $\quad$ Mild Burnout $\quad$ Moderate Burnout $\quad$ " High Burnout

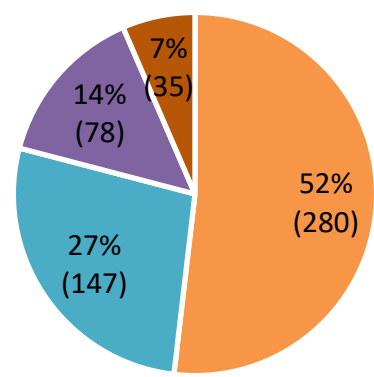

Figure 4. Participants' burnout levels.

As shown in Figure 4, the majority of participants, 51.9\%, showed normal or no burnout, while $27.2 \%$ of the participants showed mild burnout and $14.4 \%$ showed moderate burnout. Only $6.5 \%$ of participants showed a high level of burnout. 


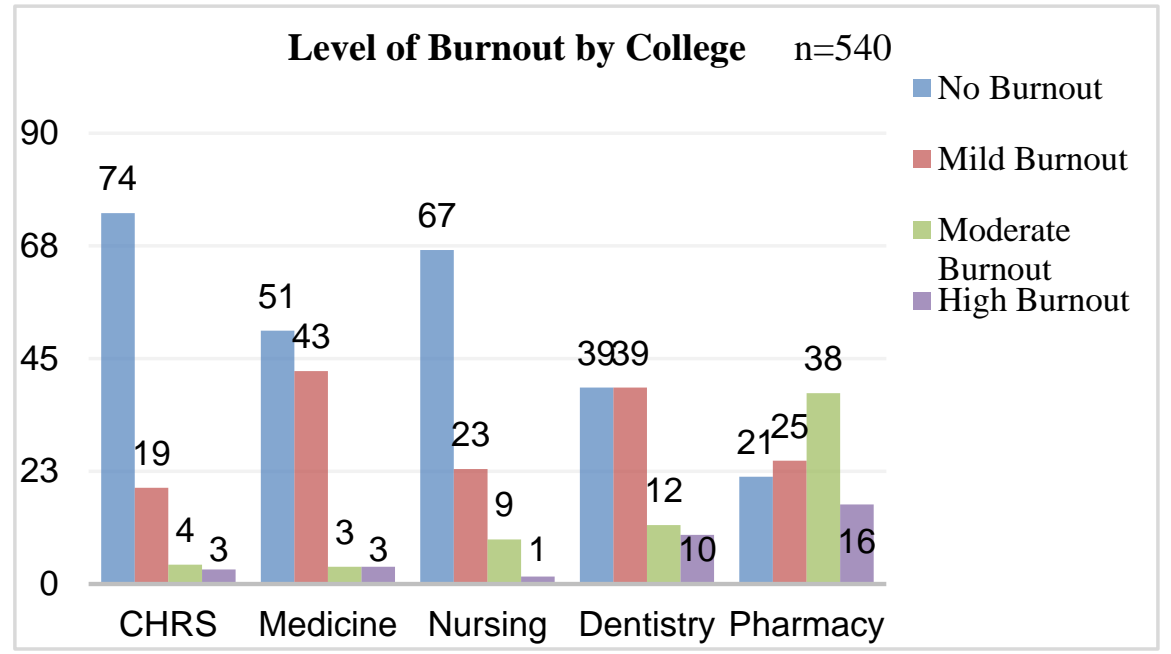

Figure 5. Level of burnout by college (p-value $=.000)$.

Burnout levels were calculated using the following categories: no burnout, low burnout, moderate burnout and high burnout. The highest levels of burnout were seen among students in the Colleges of Pharmacy, Dentistry, CHRS, Medicine and Nursing in a descending order of level of burnout. The findings of this study revealed that the levels of burnout differed across the health colleges, as the differences were associated with differences in the colleges' curricula. The majority of students who indicated high levels of burnout attended the Colleges of Pharmacy by (25.0\%) and Dentistry by (10.1).

Participants from other colleges scored low on the burnout scale. In the College of Nursing, more than two-thirds of the study participants (67\%) showed no burnout. In the College of Health and Rehabilitation Sciences, 77 out of 104 participants, more than two-thirds of the study sample, showed no burnout. The students of the College of Medicine scored low on the burnout scale where more than half of its participants $(51 \%)$ of the student sample showed no burnout at all.

\subsection{The Impact of Burnout on Students' GPA}

Table 2. Association between Burnout Categories and GPA

\begin{tabular}{lccccc}
\hline \multicolumn{5}{c}{ ANOVA BURNOUT X GPA NON SIGNIFICANT } \\
\hline Burnout Score & $\begin{array}{l}\text { Sum of } \\
\text { Squares }\end{array}$ & df & $\begin{array}{c}\text { Mean } \\
\text { Square }\end{array}$ & F & Sig. \\
Between Groups & 57.716 & 2 & 28.858 & .103 & .902 \\
Within Groups & 150406.277 & 537 & 280.086 & & \\
Total & 150463.993 & 539 & & & \\
\hline
\end{tabular}

\section{$\mathrm{P} \geq 0.05, \mathrm{df}=2$}

Table 2 shows that, overall, there is no association between the categories of burnout and GPA. Moreover, among the categories of burnout, normal burnout was the most frequently $(149,53.2 \%)$ reported. Mild burnout was also reported with medium frequency $(72,49.0 \%)$, while moderate burnout was reported with a medium level of $60 \%$ (47) of respondents. However, the high burnout category was equally rated at medium $(17,48.6 \%)$ and high levels $(17,48.6 \%)$. The overall chi-square was calculated as .399 , which shows no significance $(\mathrm{P} \geq 0.05)$.

Table 3. Association between Levels of Burnout Syndrome and Residential Status

\begin{tabular}{lllllll}
\hline $\begin{array}{l}\text { Residential } \\
\text { Status }\end{array}$ & $\begin{array}{l}\text { Frequency } \\
\text { Percentage }\end{array}$ & $\begin{array}{l}\text { Normal- } \\
\text { No } \\
\text { burnout }\end{array}$ & Mild burnout & $\begin{array}{l}\text { Moderate } \\
\text { burnout }\end{array}$ & High burnout & \multicolumn{2}{c}{$\begin{array}{c}\text { Bhi-square } \\
\text { test }\end{array}$} \\
\hline With family & Count & 260 & 132 & 70 & 32 & P-value $=.678$ \\
& $\%$ & $52.6 \%$ & $26.7 \%$ & $14.2 \%$ & $6.5 \%$ & \\
\hline
\end{tabular}




\begin{tabular}{llllll}
\hline \multirow{2}{*}{ At hostel } & Count & 20 & 15 & 8 & 3 \\
& $\%$ & $43.5 \%$ & $32.6 \%$ & $17.4 \%$ & $6.5 \%$ \\
\multirow{2}{*}{ Total } & Count & 280 & 147 & 78 & 35 \\
& $\%$ & $51.9 \%$ & $27.2 \%$ & $14.4 \%$ & $6.5 \%$ \\
\hline
\end{tabular}

As shown in Table 3, it was found that a majority of students who lived with their families showed normal burnout levels (260 participants; 52.6\%), while a smaller percentage of students who lived in a hostel (without their families) had normal levels of burnout (20 participants; $43.5 \%$ ). The total number of students who had a high level of burnout, whether they lived with their family or in a university hostel, was $35(6.5 \%)$, compared with more than half of the participants who had a normal level of burnout (280 participants; 51.9\%).

\section{Discussion}

\subsection{Demographic Characteristics of Study Participants}

The participants in this study represented five health colleges: medicine, dentistry, pharmacy, CHRS and nursing. The age range of the participants was from 19 to 26 years old, which is the ordinary age of most university students. In the KSA, children attend primary school starting at age 6 , and the education system is based on 6 years of primary school, followed by 3 years each of intermediate and secondary school; thus, it is expected that a university student will be between 18 and 23 years old (Alquaiz et al., 2012). The participants' marital and residential status revealed that most of the respondents were single and living with their families. This can be justified from a cultural perspective, as unmarried Muslim women must remain in their family homes until they get married. Generally, Muslim families in Saudi Arabia are normatively controlled by the Islamic rules and cultural values. Families are expected to comprise of a father, who is the breadwinner; and a mother, who fulfils the duties of childbearing, the household and taking care of any children who do not yet have a family.

\subsection{Levels of Burnout among Participants}

According to the participants' responses, the study found that the majority of participants had no burnout. However, the scores for the levels of burnout among females has been confirmed by several studies that females have a higher likelihood to view challenging or threatening situations as stressful, compared to males (Faris \& et al, 2016). This observation suggests that students feel better about themselves when interacting with others, which supports the view that early engagement with people is 'protective' in terms of stress and burnout (Alzahem et al., 2011). The findings of this study revealed that the levels of burnout differed across the health colleges. Most students who indicated high levels of burnout were among the Colleges of Pharmacy and Dentistry. Students of other colleges scored low in the burnout scale. The explanation for the higher levels of burnout among students in the Colleges of Pharmacy and Dentistry could be attributable to the course curriculum, which influences the level of stress and increases the burnout syndrome. A study conducted among pharmacy students to assess their level of burnout revealed that, students in pharmacy and dentistry may feel occupied with their studies and feel a sense of worth because they have chosen these specialities and at the same time they were unable to develop a resilience and to face stress and burnout due to the demands and required tasks that are put in their curriculum (Kaur et al, 2020). However, it is a necessity to determine why pharmacy and dentistry students have more burnout than other students who are studying health sciences. A qualitative investigation is required to explore more on this issue. In many studies, it has been proven that students' interests and academic satisfaction have an influence on the students' efficacy and academic performance (Polanco et al., 2014). Previous studies in the literature regarding students' burnout in the dentistry college have yielded similar results in that dental students have shown moderate to high burnout levels (Almalki, 2017 \& Albalawi, 2015). The findings in the literature regarding the level of burnout among students of the College of Medicine are unlike what was found in this study. The findings showed no significant relationship between burnout levels and medical students. Because of the lack of research regarding students' burnout in the health and rehabilitation and nursing colleges, our findings could not be compared with the previously reported studies in the literature. However, in many of the health science education colleges at PNU, students are taught via modules in which they receive information through lectures, tutorials and practical sessions. This teaching style enhances the student's skills in engaging with their peers, and any difficulties that might arise will immediately be tackled at an earlier stage of the course of the study, which helps in lowering the level of stress that increases burnout.

This study showed no significant relationship between burnout levels and students' residential status. The results also showed that 32 students (6.5\%) who live with their families had high burnout levels, while students who live in the hostel (away from their families) had low burnout levels; only three (3) students (6.5\%) had high levels of burnout. 
This result agreed with a previous study, which indicated that medical students who live with their families face more pressure in balancing their academic life and their social life, causing them to experience more emotional exhaustion and burnout. They also face high pressure from their parents to get involved in social activities that are related to family life (Al Ubaidi et al., 2018).

\subsection{Burnout and Academic Achievement}

The relationship between burnout and GPA was not significant, and no association between them was found. One explanation for this is that most of the participants were from level 8, which is the senior level of all health colleges. This indicates that students in the health colleges grow accustomed to stress and are able to cope with these stress levels because of their maturity and knowledge (Al-Kandari \& Vidal, 2007). On the other hand, the semestrial education system has an influence on the level of stress tolerance (Cuciac, Țepordei, Labăr \& Cuciac, 2015) and the students' clinical professional skills (Pitt, Powis, Levett-Jones \& Hunter, 2012). Hence, there was no association found between GPA and the high level of burnout among participants.

The findings of this study reveal that positive environment in which health sciences students are studying; paves the way of the implementation of the courses in the colleges of health sciences that promote positive behaviours and does not place students in stressful situations. This may be due to various curricular and extra-curricular activities health colleges frequently organise for their students to utilise their professional potentials, such as engaging students in community development activities to enhance their social responsibilities. In another study, it was also confirmed that higher scores for academic efficacy were correlated with lower scores for exhaustion and cynicism and higher scores in all dimensions of academic engagement. Females' self-perceptions indicated lower scores for cynicism and higher scores for academic efficacy, absorption and dedication (Liébana-Presa et al., 2018).

The results of this study revealed that there is a significant relationship between GPA levels and residential status: students who live in the university hostel had higher GPAs. Previous studies support this finding; the more time students spend on their studies and in a quiet place, the higher their GPA will be. This was confirmed by a study conducted by Onchang and Hawker (2018), who confirmed that community noise exposure and annoyance, activity interference, and academic achievement among university students affected their academic performance. Another reason might be the environment in which students live. A previous study found that on-campus students achieve higher grades than those who live off-campus. This may be because they are able to focus on their studies without distractions in their environment (Holland \& Ashley, 2014).

The present study also found a significant correlation between employment and the academic performance that was indicated by the GPAs of the participants who were employed. Two-thirds of employed students showed moderate GPAs, while $19.0 \%$ had low GPAs and $14.3 \%$ had high GPAs. Among unemployed students, two-fourths had medium GPAs, a quarter and a half had high GPAs. The study findings are in line with a study done by Tessema, Ready and Astani (2014) which showed that work had a positive effect on students' satisfaction with their GPA. However, there was no significant correlation between GPA and employment in burnout levels.

This is supported by a study conducted on King Saud University (KSU) students which found that KSU students displayed high levels of concern in private-sector work during their studies, with an average median score of 3.7 (Alasqah, 2018). This finding is also supported by Almunif's (2008) study, which found that university students have positive attitudes towards working in the private sector while studying. The attitudes of PNU students were similar, with an average of median score of 3.78. This indicates a need for real employment opportunities to be made available to students to encourage them to become involved in private-sector projects, which may help them workout and apply knowledge.

\section{Conclusions}

The findings showed that there is no association between burnout and academic achievement among students. In addition, there was no link between burnout and students' academic levels. However, there were differences in burnout levels among PNU health colleges. The study found that students in the pharmacy college were most likely to suffer from burnout compared to other health colleges.

\section{Limitations and Recommendations}

The study sample included only female students from the health colleges at PNU. Therefore, there is a gender bias. Future studies should investigate the prevalence of burnout among male students at other universities. Another limitation is that the study used a self-reporting measure for data collection, so the results may have been exaggerated. In addition, further research should be conducted to study other variables regarding students' academic achievement and other factors linked to the university context. 


\section{Acknowledgments}

This research was funded by the Deanship of Scientific Research at Princess Nourah bint Abdulrahman University through the Fast-track Research Funding Program.

\section{References}

Albalawi, A. E., Alhawiti, T. S., Aldahi, A. S., Mohammed, Y., Alshehri, S.K., \& Mirghani, H. O. (2015). The assessment of the burnout syndrome among medical students in Tabuk University, a cross-sectional analytic study. BRJMCS, 6(1), 14-19.

Al Alhareth, Y., Al Alhareth, Y., \& Al Dighrir, I. (2015). Review of Women and Society in Saudi Arabia. American Journal of Educational Research, 3(2), 121-125. https://doi.org/10.12691/education-3-2-3

Almalki, S. A., Almojali, A. I., Alothman, A. S., Masuadi, E. M., \& Alaqeel, M. K. (2017). Burnout and its association with extracurricular activities among medical students in Saudi Arabia. Int J Med Educ, 26(8), 144-150. doi: 10.5116/ijme.58e3.ca8a. PMID: 28454079; PMCID: PMC5420457. https://doi.org/10.5116/ijme.58e3.ca8a

Al Ubaidi, B., Jassim, G., \& Salem, A. (2018). Burnout Syndrome in Medical Students in the Kingdom of Bahrain. Global Journal Of Health Science, 10(11), 86. https://doi.org/10.5539/gjhs.v10n11p86

Al-asqah, A. (2018). Investigating students' attitudes towards private sector work during study in saudi arabia. Education \& Training, 60(3), 238-250. https://doi.org/10.1108/ET-05-2017-0067

Al-bakr, F., Bruce, E., Davidson, P., Schlaffer, E., \& Kropiunigg, U. (2017). Empowered but not Equal: Challenging the Traditional Gender Roles as Seen by University Students in Saudi Arabia. FIRE: Forum For International Research In Education, 4(1). https://doi.org/10.18275/fire201704011083

Aldrees, T. M., Aleissa, S., Zamakhshary, M., Badri, M., \& Sadat-Ali, M. (2013). Physician well-being: prevalence of burnout and associated risk factors in a tertiary hospital, Riyadh, Saudi Arabia. Annals of Saudi medicine, 33(5), 451-456. https://doi.org/10.5144/0256-4947.2013.451

Al-Imam, D., \& Al-Sobayel, H. (2014). The Prevalence and Severity of Burnout among Physiotherapists in an Arabian Setting and the Influence of Organizational Factors: An Observational Study. Journal Of Physical Therapy Science, 26(8), 1193-1198 https://doi.org/10.1589/jpts.26.1193

Almalki, S., Almojali, A., Alothman, A., Masuadi, E., \& Alaqeel, M. (2017). Burnout and its association with extracurricular activities among medical students in Saudi Arabia. International Journal Of Medical Education, 8, 144-150. https://doi.org/10.5116/ijme.58e3.ca8a

Alquaiz, A. M., Almuneef, M. A., \& Minhas, H. R. (2012). Knowledge, attitudes, and resources of sex education among female adolescents in public and private schools in Central Saudi Arabia. Saudi medical journal, 33(9), 1001-1009.

Al-Turki, H., Al-Turki, R., Al-Dardas, H., Al-Gazal, M., Al-Maghrabi, G., Al-Enizi, N., \& Ghareeb, B. (2010). Burnout syndrome among multinational nurses working in Saudi Arabia. Annals Of African Medicine, 9(4), 226. https://doi.org/10.4103/1596-3519.70960

Alwazzan, L., \& Rees, C. (2016). Women in medical education: views and experiences from the Kingdom of Saudi Arabia. Medical Education, 50(8), 852-865. https://doi.org/10.1111/medu.12988

Alyamani, A., Alyamani, L., Altheneyan, F., Aldhali, S., Albaker, K., \& Alshaalan, A. et al. (2018). Prevalence of Burnout among Residents at King Abdulaziz Medical City in Riyadh, Saudi Arabia. International Journal Of Medical Research \& Health Sciences, 7(12), 37-40.

Alzahem, A. M., van der Molen, H. T., Alaujan, A. H., Schmidt, G., \& Zamakhshary, M. H. (2011). Stress amongst dental students: A systematic review. Eur J Dent Educ, 15, 8-18. https://doi.org/10.1111/j.16000579.2010.00640.x

Asencio López, L., Almaraz Celis, G., Carrillo Maciel, V., Huerta Valenzuela, P., Silva Goytia, L., \& Muñoz Torres, M. et al. (2016). Burnout syndrome in first to sixth-year medical students at a private university in the north of Mexico: descriptive cross- sectional study. Medwave 2016 Abr, 16(3), e6432. https://doi.org/10.5867/medwave.2016.03.6432

Awajeh, A., Issa, M., Rasheed, A., \& Faisal Amirah, M. (2018). Burnout among Critical Care Nurses in King Saud Medical City (KSMC). Journal Of Nursing \& Care, 07(02). https://doi.org/10.4172/2167-1168.1000450

Cazan, A. (2015). Learning Motivation, Engagement and Burnout among University Students. Procedia - Social And 
Behavioral Sciences, 187, 413-417. https://doi.org/10.1016/j.sbspro.2015.03.077

Cofer, K., Hollis, R., Goss, L., Morris, M., Porterfield, J., \& Chu, D. (2018). Burnout is Associated With Emotional Intelligence but not Traditional Job Performance Measurements in Surgical Residents. Journal Of Surgical Education, 75(5), 1171-1179. https://doi.org/10.1016/j.jsurg.2018.01.021

Dafaalla, M., Bashir, S., Farah, A., \& Abdalrahman, I. (2016). Depression, Anxiety, and Stress in Sudanese Medical Students: A Cross Sectional Study on Role of Quality of Life and Social Support. American Journal of Educational Research, 26(13), 937-942. http://doi:10.12691/education-4-13-4

De Oliva Costa, E. F., Santos, S. A., de Abreu Santos, A. T. R., de Melo, E. V., \& de Andrade, T. M. (2012). Burnout Syndrome and associated factors among medical students: a cross-sectional study. Clinics, 67(6), 573-579. https://doi.org/10.6061/clinics/2012(06)05

Elbarazi, I., Loney, T., Yousef, S., \& Elias, A. (2017). Prevalence of and factors associated with burnout among health care professionals in Arab countries: a systematic review. BMC Health Services Research, 17(1). https://doi.org/10.1186/s12913-017-2319-8

Fares, J., Saadeddin, Z., Al Tabosh, H., Aridi, H., El Mouhayyar, C., Koleilat, M. K., Chaaya, M., \& El Asmar, K. (2016). Extracurricular activities associated with stress and burnout in preclinical medical students. Journal of epidemiology and global health, 6(3),177-85. https://doi.org/10.1016/j.jegh.2015.10.003

Freudenberger, H. J. (1974). Staff burn-out. Journal of Social Issues, 30, 159-165. https://doi.org/10.1111/j.15404560.1974.tb00706.x

Hamza, M., \& Abdulghani, E. (2019). Stress and Its Effects on Medical Students: A Cross-sectional Study at a College of Medicine in Saudi Arabia. Retrieved from https://www.ncbi.nlm.nih.gov/pmc/articles/PMC3225114/

Heinemann, L., \& Heinemann, T. (2017). Burnout Research: Emergence and Scientific Investigation of a Contested Diagnosis. SAGE Open, 7(1), 215824401769715. https://doi.org/10.1177/2158244017697154

Holland, \& Ashley, "How Residency Affects the Grades of Undergraduate Students" (2014). Senior Honors Theses. 85. http://digitalcommons.brockport.edu/honors/85.

IBM Corp. Released 2016, IBM SPSS Statistics For Windows, Version 24.0. Armonk, NY; IBM Corp.

Kaur, M., Long, J. W., Luk, F. S., Mar, J., Nguyen, D. L., Ouabo, T., Singh, J., Wu, B., Rajagopalan, V., Schulte, M., \& Doroudgar, S. (2020). Relationship of Burnout and Engagement to Pharmacy Students' Perception of Their Academic Ability. American journal of pharmaceutical education, 84(2), 7571. https://doi.org/10.5688/ajpe7571

Kitaoka-Higashiguchi, K., Nakagawa, H., Morikawa, Y., Ishizaki, M., Miura, K., Naruse, Y., ... Higashiyama, M. (2004). Construct validity of the Maslach Burnout Inventory-General Survey. Stress \& Health: Journal of the International Society for the Investigation of Stress, 20(5), 255-260. https://doi.org/10.1002/smi.1030

Kroska, E., Calarge, C., O’Hara, M., Deumic, E., \& Dindo, L. (2017). Burnout and depression in medical students: Relations with avoidance and disengagement. Journal Of Contextual Behavioral Science, 6(4), 404-408. https://doi.org/10.1016/j.jcbs.2017.08.003

Kulsoom, B., \& Afsar, N. A. (2015). Stress, anxiety, and depression among medical students in a multiethnic setting. Neuropsychiatric Disease and Treatment, 11, 1713-1722. https://doi.org/10.2147/NDT.S83577

Liébana-Presa, C., Fernández-Martínez, M. E., Vázquez-Casares, A. M., López-Alonso, A. I., \& Rodríguez-Borrego, M. A. (2018). Burnout and engagement among university nursing students. Enfermería Global, 17(2), 142-152. https://doi-org.sdl.idm.oclc.org/10.6018/eglobal.17.2.268831

Princess Nourah bint Abdulrahman University (2019). Retrieved from http://www.pnu.edu.sa/en/University/Pages/Intro.aspx

Richardson, M., Abraham, C., \& Bond, R. (2012). Psychological correlates of university students' academic performance: a systematic review and meta-analysis. Psychological bulletin, 138(2), 353-387. https://doi.org/10.1037/a0026838

Schonfeld, I., \& Bianchi, R. (2016). Burnout and Depression: Two Entities or One? Journal Of Clinical Psychology, 72(1), 22-37. https://doi.org/10.1002/jclp.22229

Seo, J. H., Kim, H. J., Kim, B. J., Lee, S.J., \& Bae, H. (2015). Educational and Relational Stressors Associated with Burnout in Korean Medical Students. Psychiatry Investigation, 12(4), 451-458. https://doi.org/10.4306/pi.2015.12.4.451 
Wood, B. D. (2017). Developmental students' faculty perceived levels of stress and burnout precursors among centralized and decentralized organizational layouts (Order No. 10285552). Available from ProQuest Dissertations \& Theses Global. (1940695374). Retrieved from https://search-proquestcom.sdl.idm.oclc.org/docview/1940695374?accountid=142908

Zaki, N. F. W., Denewar, K. A. F., El Sherif, M. A. F., Elweheid, A., Ibrahim, H. F., \& Pandi-Perumal, S. R. (2016). Psychological Correlates of Shift Work Sleep Disorder Among a Sample of Egyptian Nurses. Arab Journal of Psychiatry, 27(2), 127-143. https://doi.org/10.12816/0029092

\section{Copyrights}

Copyright for this article is retained by the author(s), with first publication rights granted to the journal.

This is an open-access article distributed under the terms and conditions of the Creative Commons Attribution license (http://creativecommons.org/licenses/by/4.0/). 